\title{
Charge Tunneling along Short Oligoglycine Chains
}

\section{Citation}

Baghbanzadeh, Mostafa, Carleen M. Bowers, Dmitrij Rappoport, Tomasz Zaba, Mathieu Gonidec, Mohammad H. Al-Sayah, Piotr Cyganik, Alan Aspuru-Guzik, and George M. Whitesides. 2015. "Charge Tunneling Along Short Oligoglycine Chains." Angew. Chem. Int. Ed. 54 (49) (October 9): 14743-14747. Portico. doi:10.1002/anie.201507271.

\section{Published Version}

doi:10.1002/anie.201507271

\section{Permanent link}

http://nrs.harvard.edu/urn-3:HUL.InstRepos:25013275

\section{Terms of Use}

This article was downloaded from Harvard University's DASH repository, and is made available under the terms and conditions applicable to Open Access Policy Articles, as set forth at http:// nrs.harvard.edu/urn-3:HUL.InstRepos:dash.current.terms-of-use\#OAP

\section{Share Your Story}

The Harvard community has made this article openly available.

Please share how this access benefits you. Submit a story.

\section{Accessibility}




\section{Charge Tunneling along Short Oligoglycine Chains}

Mostafa Baghbanzadeh, ${ }^{a \dagger}$ Carleen M. Bowers, ${ }^{a \dagger}$ Dmitrij Rappoport, ${ }^{a}$ Tomasz Żaba, ${ }^{b}$ Mathieu Gonidec, ${ }^{a, c}$ Mohammad H. Al-Sayah, ${ }^{a}$ Piotr Cyganik, ${ }^{b}$ Alan Aspuru-Guzik, ${ }^{a} *$ and George M. Whitesides $^{a, d, e, *}$

${ }^{a}$ Department of Chemistry and Chemical Biology, Harvard University

12 Oxford St. Cambridge, MA 02138, USA

${ }^{b}$ Smoluchowski Institute of Physics, Jagiellonian University, Łojasiewicza 11, 30-348 Krakow, Poland

${ }^{c}$ Department of Molecular Nanoscience and Organic Materials, Institut de Ciència de Materials de Barcelona (ICMAB-CSIC/CIBER-BBN), Cerdanyola del Vallès, 08193, Barcelona, Spain ${ }^{d}$ Kavli Institute for Bionano Science and Technology, Harvard University

29 Oxford Street, Cambridge, MA 02138, USA

${ }^{e}$ Wyss Institute of Biologically Inspired Engineering, Harvard University 60 Oxford St. Cambridge, MA 02138, USA

${ }^{\dagger}$ Both authors contributed equally to this work.

*To whom correspondence may be addressed: gwhitesides@gmwgroup.harvard.edu, and aspuru@,chemistry.harvard.edu 


\section{Abstract}

Charge transport through self-assembled monolayers (SAMs) of oligoglycines (Gly, $\mathrm{n}=0-5)$ having a cysteine (Cys) group that anchors the molecule to a gold substrate is rapid (relative to SAMs of $n$-alkanethiolates). Comparisons of rates of charge transport-using junctions with the structure $\mathrm{Au}^{\mathrm{TS}} / \mathrm{SAM} / / \mathrm{Ga}_{2} \mathrm{O}_{3} / \mathrm{EGaIn}$ - across these SAMs of oligoglycines, and across SAMs of a number of related molecules - established that rates of charge tunneling along SAMs of oligoglycines are comparable to that along SAMs of oligophenyl groups (of comparable length). The mechanism of tunneling in oligoglycines is compatible with superexchange, and involves interactions among high-energy occupied orbitals in multiple, consecutive amide bonds, which may by separated by one to three methylene groups. This mechanistic conclusion is supported by density functional theory (DFT).

Keywords: Tunneling; charge transport; superexchange; oligopeptides; biological conduction; organic electronics; SAM; DFT 
Understanding mechanisms of charge transport (CT) in proteins is important, both for biology and nanoscience.(1-6) Self-assembled monolayers (SAMs) of oligopeptides offer the opportunity to investigate charge transport across biologically relevant model structures (and to examine molecular entities present in proteins).( $(1,3,7) \mathrm{CT}$ is fundamental (for example) to photosynthesis, $(4,8)$ some enzymatic reactions(9) and respiration,(4) and can be rapid over considerable distances. $(10,11)$ The mechanisms (e.g., tunneling, inelastic hopping, or other processes) of CT through peptides are still incompletely defined, and have been variously suggested to depend on the length of the peptide, the presence of secondary structure, and the presence of specific side chains. $(1,12,13)$

Rates of charge tunneling across SAMs composed of $n$-alkanethiolates have been studied extensively (14-18), and most commonly characterized using the parameters of the simplified Simmons equation (eq. 1).(19) This equation is a useful (and commonly used) semi-empirical parameterization that suggests that the rate of tunneling should depend exponentially on the width of a barrier (d), assumed to be rectangular, and on $\beta$ (a parameter related to the height of the barrier).

$$
J(V)=J_{0}(V) \mathrm{e}^{-\beta d}=J_{0}(V) 10^{-\beta d / 2.303}
$$

Values of $\beta$ for $n$-alkanethiolates are $\beta_{S C n}=0.9-1.1 \mathrm{n}_{\mathrm{CH} 2}{ }^{-1}$ or $\beta_{S C n}=0.7-0.8 \AA^{-1}$.(18) The rates of charge transport across SAMs structurally more complex than linear hydrocarbons (i.e., SAMs with aryl groups, with heteroatoms embedded in the backbone, and SAMs with the potential for strong interchain interactions) have also been explored $\left(\beta_{(P h) n}=0.3-0.6 \AA^{-1}\right) \cdot(20-24)$

This work characterized the rates of CT across SAMs containing oligoglycine residues, and compared these rates with those across $n$-alkanethiolates of approximately matched length. Its objectives were to determine i) if oligopeptides provided a better pathway for $\mathrm{CT}$ by 
tunneling than did $n$-alkanes and ii) if there were a difference between oligoamides and $n$ alkanes, to suggest, both experimentally and theoretically, why the two behaved differently.

We investigated CT across SAMs of six compositionally simple derivatives of oligoglycine (Fig. 1) with a terminal cysteine residue $\left(\mathrm{Cys}(\mathrm{Gly})_{\mathrm{n}}, \mathrm{n}=0-5\right)$, using a large-area junction having the structure $\mathrm{Au}^{\mathrm{TS}} / \mathrm{SAM} / / \mathrm{Ga}_{2} \mathrm{O}_{3} / \mathrm{EGaIn}$, where $\mathrm{Au}^{\mathrm{TS}}$ is a template-stripped gold surface.(18) (This junction has been described in previous papers.(18, 20, 25-28)) SAMs having oligoglycine residues gave values of $\beta$ that are significantly lower than those measured for SAMs of $n$ alkanethiolates of similar length. DFT modeling of gold-bound oligoglycines (below) indicates that the high-energy occupied orbitals of peptide groups, which modulate the local tunneling potential, are essential for rapid tunneling. Although the exponential length dependence of tunneling rates in $n$-alkanethiols can be approximated by a flat, rectangular tunneling barrier (Eq. (1)), the tunneling conductance through chains of oligopeptides is better described by a onedimensional chain of peptide groups coupled electronically. This model was termed superexchange tunneling by McConnell, Ratner, Nitzan, and co-workers using an analogy with reaction kinetics of processes involving CT,(29-31) and was used not only as a model for CT in peptides $(4,7,32)$, but also as a simple qualitative model for molecular conduction. $(29,30,33)$ Here, we establish that the superexchange model can be used to interpret experimental tunneling data, by correlating measurements of current density and calculations of electronic structure. We use the superexchange model to derive the relevant model parameters from DFT, and demonstrate that this model is applicable to amide groups separated by methylene groups $\left(\left(\mathrm{CH}_{2}\right)_{\mathrm{n}}\right.$ with $\left.\mathrm{n}=1-3\right)$. Notably, both the flat-barrier and the superexchange models predict an exponential length dependence in tunneling rates.(19) 
SAMs on template-stripped gold $\left(\mathrm{Au}^{\mathrm{TS}}\right)$ were prepared according to protocols reported previously. $(18,25)$ Detailed surface analyses indicate that SAMs of Cys $(\mathrm{Gly})_{\mathrm{n}}$ are ordered: Infrared absorption-reflection spectroscopy (IRRAS) data indicates that SAMs of oligoglycine form beta-sheet structures and X-ray photoelectron spectroscopy (XPS) and ellipsometry measurements confirm that the thickness of these SAMs grows linearly with an increase in the number of glycine residues. The supporting information contains details on the formation and characterization of the SAMs, of the measurements, and of the DFT calculations.

We derive values of $\beta$ and $J_{0}$ (eq. 1) for oligopeptides on gold (Cys(Gly $)_{\mathrm{n}}, \mathrm{n}=0-5$ ), and compare these values with those of $n$-alkanethiolates, to determine the influence of the differences in their structure on the rates of charge transport by tunneling across them. Linear regression of $\log |J|$ versus $\mathrm{n}$ (the number of non-hydrogen atoms in the backbone) for SAMs of oligoglycine on $\mathrm{Au}^{\mathrm{TS}}$ yielded $\beta_{(G l y) n}=0.5 \pm 0.02 \mathrm{n}_{\text {atom }}{ }^{-1}$ and $\log \left|J_{0(\mathrm{Gly}) \mathrm{n}}\right|=2.8 \pm 0.3$ (Fig. 2, S7, and S11). Measurements of $J(\mathrm{~V})$ for all peptides resulted in symmetric voltage profiles at forward and reverse biases $( \pm 0.5 \mathrm{~V})$; none showed a rectification ratio $(r=$ $|J(+0.5 \mathrm{~V})| /|J(-0.5 \mathrm{~V})|)$ larger than 3 (similar to SAMs of alkanethiolates $(18)$ ). The difference between $\log \left|J_{0}\right|$ for SAMs of oligoglycines $\left(\log \left|J_{0(\mathrm{Gly}) n}\right|=2.8 \pm 0.3\right)$, and $n$-alkanethiolates, both on gold $\left(\log \left|J_{0 S C n}\right|=3.6 \pm 0.3\right)$, was statistically significant, as was the difference between the value of $\beta\left(\beta_{(G l y) n}=0.50 \pm 0.02 \mathrm{n}_{\text {atom }}{ }^{-1}, \beta_{S C n}=0.94 \pm 0.02 \mathrm{n}_{\mathrm{C}}^{-1}\right)$. (The $\log \left|J_{0}\right|$ for $n$-alkanethiolates on gold in units of $\AA^{-1}$ is $\left.\sim 4 .(25,34)\right)$ The value of $J_{0}$ is well-defined experimentally, but requires an extrapolation in the data that makes its interpretation ambiguous; we explain this ambiguity in the supplementary materials.

SAMs of oligoglycines (Fig. 1,2) are more conductive by tunneling than SAMs of alkanethiolates. To explain this difference, we tested two hypotheses: i) $3 D$ structure. The 
presence of an extended network of hydrogen bonds connecting the (Gly $)_{n}$ groups intermolecularly might result in changes in molecular or electronic structure of the junction that would influence the rates of charge transport. ii) The effect of interactions between amide bonds. The presence of multiple, interacting amide bonds along the chain might increase tunneling by a superexchange tunneling mechanism involving a sequence of high-lying coupled orbitals.(29, 30, 35) To examine the effect of hydrogen bonds in charge transfer we compared the current density across Cys $(\mathrm{Gly})_{3}$ and cysteine trisarcosine $\left(\mathrm{Cys}(\mathrm{Sar})_{3},(\mathbf{1 9})\right)$. Substituting a methyl group for a hydrogen atom on the nitrogen atom of the amide groups - a change that converts $\mathrm{Cys}(\mathrm{Gly})_{3}$ to $\mathrm{Cys}(\mathrm{Sar})_{3}$ - eliminates the possibility of inter-chain hydrogen-bond formation, and has the potential to influence the conformation of the SAM. Although we have not explicitly addressed the issue of structure in detail, we know from measurements using spectroscopic ellipsometry that both SAMs of Cys(Gly) ${ }_{3}$ and SAMs of Cys(Sar) ${ }_{3}$ have comparable thicknesses (Fig. S4B)). Measurements of current density for these two compounds yielded indistinguishable values of $\log |J|$ for $\operatorname{Cys}(\mathrm{Gly})_{3}(0.0 \pm 0.4)$ and $\operatorname{Cys}(\operatorname{Sar})_{3}(0.0 \pm 0.6)$; this similarity strongly suggests that the tunneling current, in this system, is not influenced by hydrogen bonds or changes in conformation (Fig. 3A and S9).

To check the hypothesis that orbital interaction between the amide bonds are important, we compared the tunneling current between two sets of molecules (4 and 12-14, and 15-18). In each set, the number of amide bonds increased sequentially; the difference between the two series is the presence of an amine functional group on cysteine. Fig. 3 summarizes the results of this study. This analysis shows that increasing the number of amide bonds within molecules of the same length increases their conductivity (Fig. 3). 
DFT calculations on the oligoglycines (see the supplemental material for details), and their analogs, indicate that each amide group contributes two high-lying occupied orbitals; these orbitals arise from the lone pairs on oxygen and nitrogen. In the all-trans conformation, these orbitals are distinguishable by their symmetry; that is, they are either in plane or perpendicular to the plane of the amide group (Fig. 4A and 4B). For an isolated amide bond of CysGly, the orbital energies of the lone pairs on oxygen and nitrogen are found at ca. -6.6 and $-7.0 \mathrm{eV}$. Due to symmetry, only interactions within groups of "in-plane" or "out-of-plane" occupied orbitals are possible. These sets generate two independent manifolds of high-lying occupied orbitals with energies between -6 and $-8 \mathrm{eV}$. We observe that the orbital energies within each manifold follow a pattern consistent with the predictions of the superexchange tunneling model, which assumes a linear chain of localized orbitals with nearest-neighbor interactions. (29, 30, 35) (Fig. 4C) In turn, the superexchange model predicts that large nearest-neighbor couplings lead to rapid tunneling rates, as observed experimentally in measurements of current density across SAMs of oligoglycine. The strength of the interaction is sensitive to the relative orientation of the amide groups and is influenced by the (yet unknown) details of the molecular conformation. The supplementary materials describe these DFT calculations.

To determine how the distance between the amide bonds influences CT, we measured current density across increasing lengths of cysteine- $\beta$-alanine (compounds 22-24). These structures have two $\mathrm{CH}_{2}$ groups between the amide bonds, compound $\mathbf{2 5}$ has three $\mathrm{CH}_{2}$ groups between the amide bonds, and compound 26 has four $\mathrm{CH}_{2}$ groups. The data in Fig. 3B show that four $-\mathrm{CH}_{2}-$ units between the amide groups are necessary to reduce the tunneling current density to a value close to a length-matched $n$-alkanethiolate. This finding is consistent with the computed nearestneighbor interactions between amide groups obtained from DFT calculations. Fitting of the DFT 
occupied orbital energies (Fig. 4B) to the superexchange model shows that the strength of nearest-neighbor interactions between the in-plane occupied orbitals in the compounds $\mathbf{2 2 - 2 5}$ is similar to that in oligoglycines.

A comparison of $\log |J|$ values between compound $10(\log |J|=1.9 \pm 0.6)$ and Cys $(\mathbf{1})(\log |J|=$ $1.9 \pm 0.4)$, and $4\left(\mathrm{Cys}(\mathrm{Gly})_{3},-0.1 \pm 0.4\right)$ and compound $20(0 \pm 0.3)$ confirm that the amine group does not influence the tunneling current (Fig. 2 and 3). Measurements of current density through compounds 20, 21, and $\mathbf{1 8}$ showed that changing the terminal $-\mathrm{COOH}$ group in $\mathbf{2 0}(\log |J|=$ $0 \pm 0.3)$ to $-\mathrm{COOMe}$ in $21(\log |J|=-0.4 \pm 0.3)$ or $-\mathrm{CH}_{2} \mathrm{CH}_{3}$ in $\mathbf{1 8}(\log |J|=-0.4 \pm 0.3)$ does not influence $\mathrm{CT}$ beyond the small difference $(\Delta \log |J|=0.4)$ expected for an additional carbon.

This study of charge transport across short sequences of oligoglycines results in five key conclusions. i) Tunneling is the dominant mechanism for CT through short sequences of oligoglycines. The results indicate that charge transport by tunneling occurs across SAMs of oligoglycines having the structure of CysGly $($ with $n=0-5$ ); there was no apparent change in the mechanism of CT (e.g. from tunneling to hopping) within this series of compounds. ii) SAMs of oligoglycines are more conductive by tunneling than SAMs of alkanethiolates. Tunneling current densities across increasing lengths of oligoglycines in SAMs on gold showed a lower value of $\beta$ $\left(\beta_{(G l y) n}=0.50 \pm 0.02 \mathrm{n}_{\text {atom }}^{-1}\right)$ than that measured for SAMs of $n$-alkanethiolates $\left(\beta_{S C n}=0.94 \pm 0.02\right.$ $\mathrm{n}_{\mathrm{C}}^{-1}$ ) (Fig. 2). This differences can be large for long chains (for instance

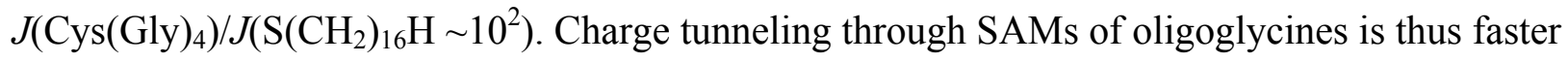
than that through SAMs of alkanethiolates of the same length, and that difference becomes larger as the chains become longer. iii) The presence of multiple high-lying, occupied, interacting amide orbitals is responsible for the high conductivity of oligoglycines. iv) Superexchange by this mechanism plays a dominant role in CT, and non-nearest-neighbor coupling between in- 
plane orbitals is possible. Surprisingly (but supported by calculations) -

$(\mathrm{CONH})\left(\mathrm{CH}_{2}\right)_{\mathrm{n}}(\mathrm{CONH})-($ with $\mathrm{n}=1-3)$ all enhance the rates of tunneling relative to the lengthmatched $\left(\mathrm{CH}_{2}\right)_{\mathrm{n}}$ groups. v) The rate of CT by tunneling through oligoglycines is comparable with linear polyaromatics. The attenuation of the rate of CT by tunneling through SAMs of (Gly) $)_{n}$ is comparable with values of $\beta\left(\beta_{(G l y) n}=0.45 \pm 0.02 \AA^{-1}\right)$ reported for linear polyaromatics $\left(\beta_{(P h) n}=\right.$ $\left.0.3-0.6 \AA^{-1}\right) \cdot(23,24)$

The superexchange tunneling model predicts that two structural factors are responsible for rapid hole tunneling through oligoglycine chains relative to $n$-alkanethiolates: i) the high-lying occupied orbitals on the amide bonds, and ii) the substantial nearest-neighbor couplings between them. This insight provides us with useful design strategy for new tunneling conductors based on maximizing the nearest-neighbor couplings between high-lying occupied orbitals in organic molecules.

\section{Acknowledgements}

This work was supported by a subcontract from Northwestern University from the United States Department of Energy (DOE, DE-SC0000989). The DOE grant from Northwestern also supported the salary for M.B. and C.M.B. M. G. acknowledges support from Marie Curie IOF FP7 for project SAM-TunEGaIn (PIOF-GA-2012-328412). M.A. thanks his institution (FRG1302-04), the Arab Fund (2-1-72/1841) and ONR Global (N62909-14-1-V17) for financial support.

We thank Dr. Kyungtae Kang for assistance in ellipsometry analysis. D.R and A. A.-G. acknowledge the Cyberdiscovery Initiative Type II (CDI2) grant from the National Science

Foundation (NSF, OIA-1125087). T. Z. and P. C. acknowledge the National Science Centre Poland (grant DEC-2013/10/E/ST5/00060).

\section{Supplementary Materials}

Materials and Methods 
Supplementary Text

Figs. S1 to S14

Schemes $\mathrm{S} 1$ to $\mathrm{S} 3$

Tables S1 to S5

\section{References and Notes}

1. A. Shah et al., Electron transfer in peptides. Chem. Soc. Rev. 44, 1015 (2015).

2. M. W. Shinwari, M. J. Deen, E. B. Starikov, G. Cuniberti, Electrical conductance in biological molecules. Adv. Funct. Mater. 20, 1865 (2010).

3. S. Sek, Peptides and proteins wired into the electrical circuits: An SPM-based approach. Biopolymers 100, 71 (2013).

4. M. Cordes, B. Giese, Electron transfer in peptides and proteins. Chem. Soc. Rev. 38, 892 (2009).

5. J. R. Winkler, H. B. Gray, Long-range electron tunneling. J. Am. Chem. Soc. 136, 2930 (2014).

6. I. Ron, I. Pecht, M. Sheves, D. Cahen, Proteins as solid-state electronic conductors. Acc. Chem. Res. 43, 945 (2010).

7. Y.-T. Long, E. Abu-Irhayem, H.-B. Kraatz, Peptide electron transfer: More questions than answers. Chem. Eur. J. 11, 5186 (2005).

8. S. Eberhard, G. Finazzi, F. A. Wollman, The dynamics of photosynthesis. Annu. Rev. Genet. 42, 463 (2008).

9. J. Stubbe, D. G. Nocera, C. S. Yee, M. C. Y. Chang, Radical initiation in the class I ribonucleotide reductase: Long-range proton-coupled electron transfer? Chem. Rev. 103, 2167 (2003).

10. Y. Arikuma, H. Nakayama, T. Morita, S. Kimura, Electron hopping over 100 angstrom along an alpha helix. Angew. Chem. Int. Ed. 49, 1800 (2010). 
11. B. Giese, M. Graber, M. Cordes, Electron transfer in peptides and proteins. Curr. Opin. Chem. Biol. 12, 755 (2008).

12. Y. Arikuma, K. Takeda, T. Morita, M. Ohmae, S. Kimura, Linker effects on monolayer formation and long-range electron transfer in helical peptide monolayers. J. Phys. Chem. B 113, 15900 (2009).

13. N. Amdursky et al., Electronic transport via proteins. Adv. Mater. 26, 7142 (2014).

14. A. Salomon et al., Comparison of electronic transport measurements on organic molecules. Adv. Mater. 15, 1881 (2003).

15. R. L. McCreery, Molecular electronic junctions. Chem. Mater. 16, 4477 (2004).

16. R. L. McCreery, A. J. Bergren, Progress with molecular electronic junctions: meeting experimental challenges in design and fabrication. Adv. Mater. 21, 4303 (2009).

17. J. P. Bergfield, M. A. Ratner, Forty years of molecular electronics: Non-equilibrium heat and charge transport at the nanoscale. Phys. Status Solidi B 250, 2249 (2013).

18. F. C. Simeone et al., Defining the value of injection current and effective electrical contact area for EGaln-based molecular tunneling junctions. J. Am. Chem. Soc. 135, 18131 (2013).

19. J. G. Simmons, Generalized formula for electric tunnel effect between similar electrodes separated by a thin insulating film. J. Appl. Phys. 34, 1793 (1963).

20. M. M. Thuo et al., Replacing - $\mathrm{CH}_{2} \mathrm{CH}_{2}$ - with - $\mathrm{CONH}$ - does not significantly change rates of charge transport through $\mathrm{Ag}^{\mathrm{TS}}-\mathrm{SAM} / / \mathrm{Ga}_{2} \mathrm{O}_{3} / \mathrm{EGaIn}$ junctions. J. Am. Chem. Soc. 134, 10876 (2012).

21. H. J. Yoon, C. M. Bowers, M. Baghbanzadeh, G. M. Whitesides, The rate of charge tunneling is insensitive to polar terminal groups in self-assembled monolayers in 
$(\mathrm{AgS})^{\mathrm{TS}}\left(\mathrm{CH}_{2}\right)(\mathrm{n}) \mathrm{M}\left(\mathrm{CH}_{2}\right)(\mathrm{m}) \mathrm{T} / / \mathrm{Ga}_{2} \mathrm{O}_{3} / \mathrm{EGaIn}$ junctions. J. Am. Chem. Soc. 136, 16 (2014).

22. A. Haj-Yahia et al., Substituent variation drives metal/monolayer/semiconductor junctions from strongly rectifying to ohmic behavior. Adv. Mater. 25, 702 (2013).

23. B. Kim, S. H. Choi, X. Y. Zhu, C. D. Frisbie, Molecular tunnel junctions based on $\pi$ conjugated oligoacene thiols and dithiols between $\mathrm{Ag}, \mathrm{Au}$, and Pt contacts: effect of surface linking group and metal work function. J. Am. Chem. Soc. 133, 19864 (2011).

24. T. Toledano et al., Odd-even effect in molecular electronic transport via an aromatic ring. Langmuir 30, 13596 (2014).

25. C. M. Bowers et al., Characterizing the metal-SAM interface in tunneling junctions. ACS Nano 9, 1471 (2015).

26. L. Jiang, L. Yuan, L. Cao, C. A. Nijhuis, Controlling leakage currents: The role of the binding group and purity of the precursors for self-assembled monolayers in the performance of molecular diodes. J. Am. Chem. Soc. 136, 1982 (2014).

27. L. Yuan, L. Jiang, D. Thompson, C. A. Nijhuis, On the remarkable role of surface topography of the bottom electrodes in blocking leakage currents in molecular diodes. $J$. Am. Chem. Soc. 136, 6554 (2014).

28. L. Yuan, L. Jiang, B. Zhang, C. A. Nijhuis, Dependency of the tunneling decay coefficient in molecular tunneling junctions on the topography of the bottom electrodes. Angew. Chem. Int. Ed. 53, 3377 (2014).

29. A. Nitzan, Electron transmission through molecules and molecular interfaces. Annu. Rev. Phys. Chem. 52, 681 (2001). 
30. R. Kosloff, M. A. Ratner, Superexchange-assisted through-bridge electron-transferelectronic and dynamic aspects. Israel J. Chem. 30, 45 (1990).

31. H. McConnell, Intramolecular charge transfer in aromatic free radicals. J. Chem. Phys. 35, 508 (1961).

32. H. B. Gray, J. R. Winkler, Long-range electron transfer. Proc. Natl. Acad. Sci. USA 102, 3534 (2005).

33. C. Joachim, M. A. Ratner, Molecular electronics: Some views on transport junctions and beyond. Proc. Natl. Acad. Sci. USA 102, 8801 (2005).

34. M. Baghbanzadeh et al., Odd-even effects in charge transport across n-alkanethiolatebased SAMs. J. Am. Chem. Soc. 136,16919 (2014).

35. J. A. Hoerni, Application of free-electron theory to three-dimensional networks. J. Chem. Phys. 34, 508 (1961). 


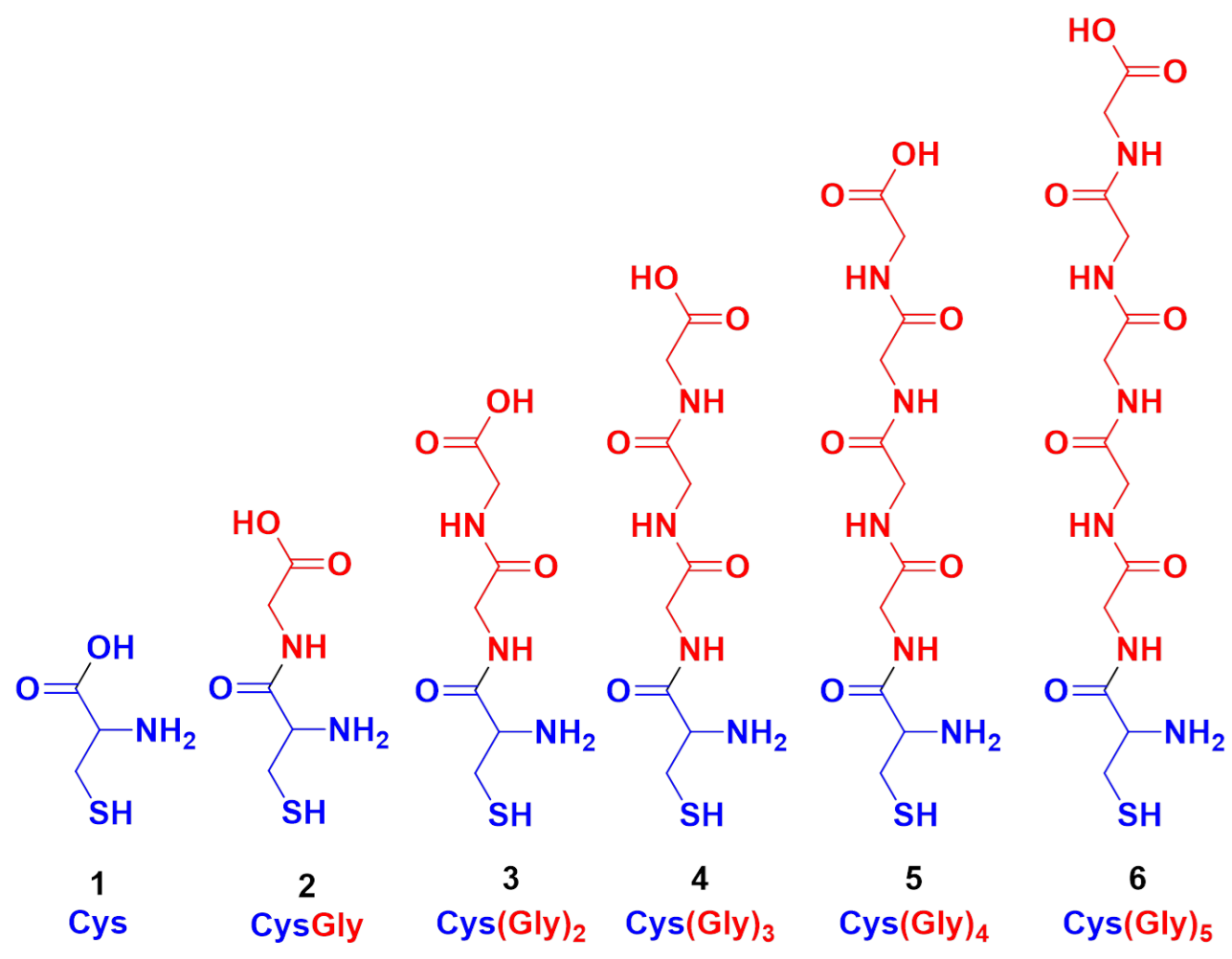

Fig.1. Structures of the investigated series of cysteine-glycines $\left(\mathrm{Cys}(\mathrm{Gly})_{\mathrm{n}}\right)$ peptides investigated in this work. 


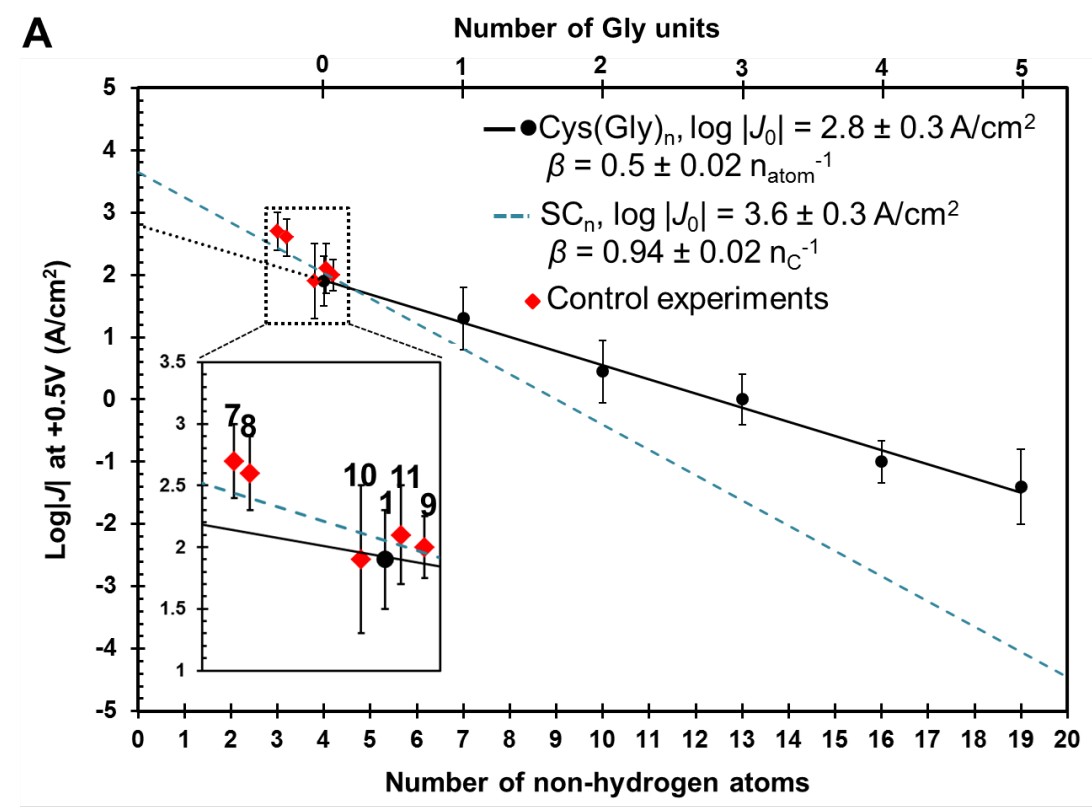

B

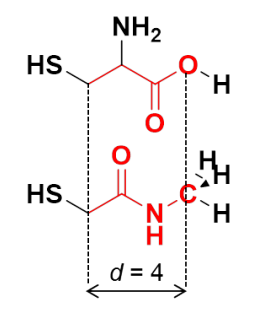

C

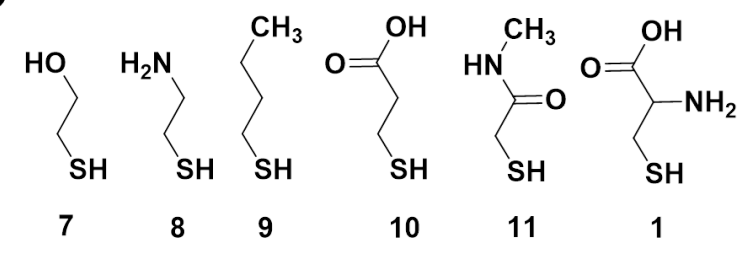

Fig.2. A) Plot of the Gaussian mean values of $\log |J|$ at $+0.5 \mathrm{~V}$ versus molecular length for oligoglycines (solid line) and $n$-alkanethiolate standards (dashed line) using a junction with the structure $\mathrm{Au}^{\mathrm{TS}} / \mathrm{SAM} / / \mathrm{Ga}_{2} \mathrm{O}_{3} / \mathrm{EGaIn}$. Error bars represent the standard deviation of Gaussian mean values. Molecules that are shorter than cysteine (1), but still possess some structural similarities to it $(\mathbf{7}, \mathbf{8})$, give current densities that fit to the line for $n$-alkanethiolates (dashed line). B) The molecular length is calculated based on the number of non-hydrogen atoms, starting with carbon next to the sulfur atom, and counting to the final non-hydrogen atom (for example, number of atoms for both Cys (1) and $\mathbf{1 1}$ is 4). C) Structure of "short" molecules that are similar in composition to cysteine. The current densities of these molecules were measured to develop a feeling for the reliability of extrapolation as a method of estimating $J_{0}$ for the series. $\mathrm{Cys}(\mathrm{Gly})_{\mathrm{n}} \mathrm{OH}$. 

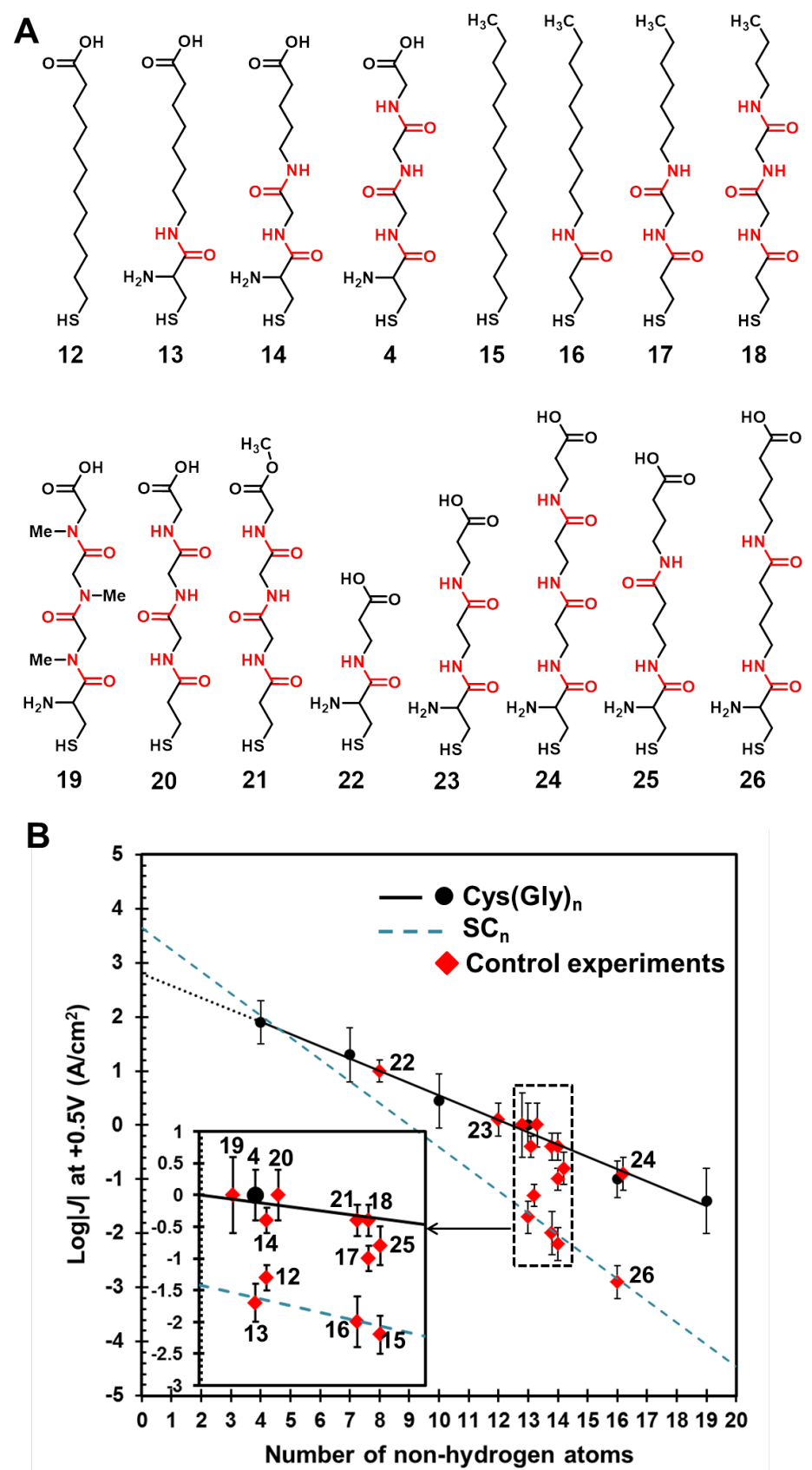

Fig.3. A) Structure of the compounds used for control experiments. B) Influence of an amide bond on current density. The solid line represents the fit for Gaussian mean values of $\log |J|$ at $+0.5 \mathrm{~V}$ for oligoglycines, and the dashed line represents the line for $n$-alkanethiolate on $\mathrm{Au}^{\mathrm{TS}}$. The numbers corresponds to compounds in Fig. 3A. The molecular length is calculated based on the number of non-hydrogen atoms from the sulfur atom to the final atom. Error bars represent standard deviation of Gaussian mean value. 

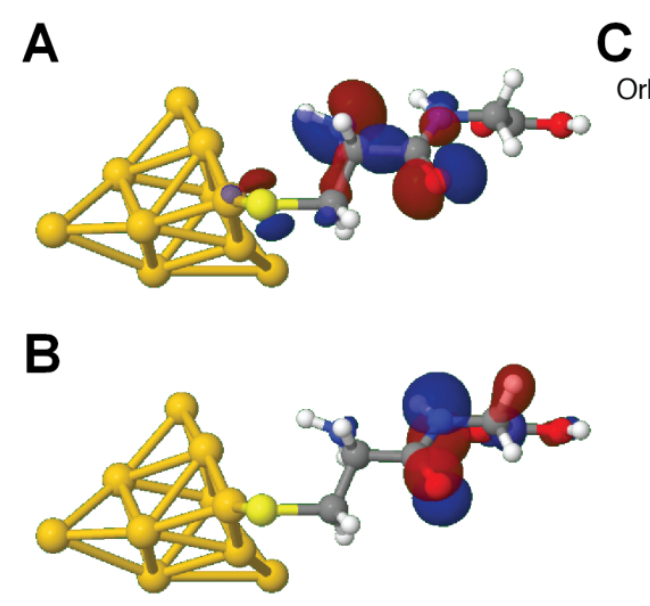

Orbital Energy, eV

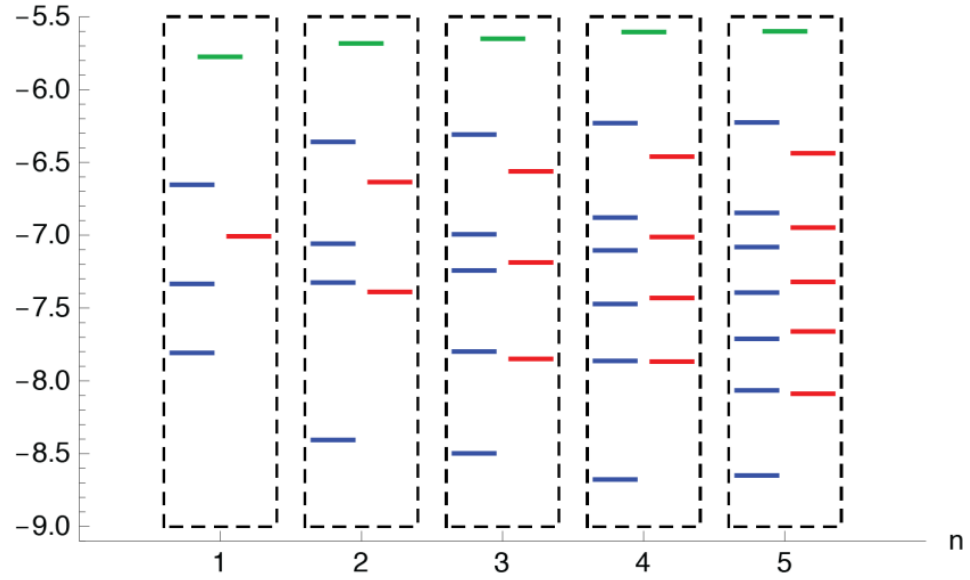

Fig.4. Relevant high-lying occupied orbitals of cysteine-glycines (Cys $\left.(\mathrm{Gly})_{\mathrm{n}}\right)$ peptides from DFT calculations. A) In-plane occupied orbital of the peptide bond. B) Out-of-plane occupied orbital of the peptide bond. C) Orbital energies of the sulfur lone pair orbitals (green), in-plane (blue), and out-of-plane (red) occupied orbitals of the amide bonds in oligoglycine complexes $\left(\mathrm{Au}_{10^{-}}\right.$ $\left.\operatorname{Cys}(\mathrm{Gly})_{\mathrm{n}}\right)$ peptides as function of chain length $\mathrm{n}$. 